\title{
The EvoS Consortium: Catalyzing Evolutionary Training in Higher Education
}

\author{
Introduction to this Special Issue Devoted to the EvoS Consortium
}

\author{
David Sloan Wilson • Glenn Geher • Jennifer Waldo • \\ Rosemarie Sokol Chang
}

Published online: 8 February 2011

(C) Springer Science+Business Media, LLC 2011

\begin{abstract}
EvoS is a consortium of evolutionary studies programs that can catalyze evolutionary training across the curriculum in higher education. This special issue of Evolution: Education and Outreach shows how the dictum "Nothing in biology makes sense except in the light of evolution" can be achieved within the biological sciences and expanded to include all human-related subjects, both for undergraduate education and faculty interactions, fulfilling the ideal of a liberal arts education and turning the university into a single intellectual community.
\end{abstract}

Keywords EvoS · EvoS Consortium · Evolution · Education $\cdot$ Evolutionary studies

\author{
D. S. Wilson $(\bowtie)$ \\ Departments of Biology and Anthropology, \\ Binghamton University, \\ Binghamton, NY, USA \\ e-mail: dwilson@binghamton.edu \\ URL: http://evostudies.org/ \\ G. Geher $\cdot$ R. S. Chang \\ Department of Psychology, SUNY New Paltz, \\ New Paltz, NY, USA \\ G. Geher \\ e-mail: geherg@newpaltz.edu \\ URL: http://evostudies.org/ \\ R. S. Chang \\ e-mail: rischang@gmail.com \\ URL: http://evostudies.org/ \\ J. Waldo \\ Department of Biology, SUNY New Paltz, \\ New Paltz, NY, USA \\ e-mail: waldoj@newpaltz.edu \\ URL: http://evostudies.org/
}

A catalyst is a substance that can vastly increase the rate of a reaction, even when added in small amounts, without being used up in the process. Catalysts perform a kind of miracle. In their absence, the reaction takes place slowly or not at all. When added, that which was difficult before becomes easy.

Cultural reactions can be catalyzed in addition to chemical reactions. Consider the acceptance of evolution in various sectors of society. It has become the overarching theoretical framework for the biological sciences, enabling Theodosius Dobzhansky (1973) to say-almost forty years ago - that "nothing in biology makes sense except in the light of evolution." Yet, a close look at the biological sciences reveals that acceptance of evolution is uneven. Few biologists deny evolution, but many fail to use it when conducting their research and teaching their courses. If Dobzhansky's dictum was really taken seriously, then evolution would be the first thing that college students learn in their introductory courses and would organize the rest of the biology curriculum-for cell and molecular biology no less than ecology and behavior. Evolution would provide the theoretical framework for premedical education and the medical school curriculum. Some biology curricula are exemplary in this regard, but many others fall short (Alters and Nelson 2002; Gluckman et al. 2009).

As we move from the biological sciences to the traditionally human-related disciplines - which means most departments on a college campus - the situation gets worse. When Darwin first proposed his theory, it was obvious to everyone that, if true, it would have momentous consequences for our understanding of ourselves. Yet, by the early twentieth century, the study of evolution was largely restricted to the biological sciences and avoided for most human-related subjects, especially concerning human be- 
havior and culture. A common formulation has been that evolution explains the rest of life, our physical bodies, and a few basic urges such as to eat and have sex, but has nothing to say about our rich behavioral and cultural diversity (Wilson 2005a). It is important to understand the complex history of ideas that caused this formulation to arise, but the bottom line is that the average professor of psychology, sociology, cultural anthropology, history, economics, political science, or literature is unlikely to have received any evolutionary training during their own higher education and therefore unlikely to teach it to his or her students.

As we move from higher education to childhood education and acceptance of evolution by the general public, the situation gets still worse. America's denial of evolution is well known, but nearly everyone everywhere who "accepts" evolution fails to think about it in relation to what matters in their lives, either professionally or personally (Wilson 2007).

In short, the acceptance of evolution provides an outstanding example of a cultural reaction taking place slowly or not at all. If it is possible to catalyze a cultural reaction, similar to a chemical reaction, then we want to know about it. That which was difficult before would become easy. We're happy to report that there is such a catalyst and it is already working its magic. It is a consortium of evolutionary studies programs called EvoS, which is showcased in this special issue of Evolution: Education and Outreach (EEO).

\section{Ingredients of EvoS}

Thanks largely to a grant from the National Science Foundation (Award no. 0817337), the EvoS Consortium has its own website (http://evostudies.org/) and its own open-access journal (http://evostudies.org/evos-journal/ about-the-journal/), providing the resources for any individual or organization to become involved. This special issue of $E E O$ provides a glimpse of what it means to take Dobzhansky's dictum seriously across the entire college curriculum - including mainstream evolutionary biology (Halverson), premedical education (Waldo and Greagor), psychology (Geher, Crosier, Dillon, and Chang), family studies and human development (King and deBaca), childhood education (Gray), environmental studies and literature (Hart and Long), the mass media (Fisher, Kruger, and Garcia) nutrition and physical fitness (Platek, Geher, Heywood, Stapell, Porter, and Waters), general education pedagogy (O'Brien and Gallup; Price), and involving undergraduate students in the peer-review process (Chang).

The goals of the EvoS Consortium and EEO are one and the same, which makes this special issue a great moment for both entities. EvoS and EEO are both predicated on the facts that (a) understanding evolution is crucial for understanding the nature of virtually all living phenomena and (b) improving evolution education should be a primary goal in thinking about how to shape educational systems writ large.

In this introduction to the special issue, we describe how the EvoS Consortium functions as a catalyst in a matter-offact sense. After all, it does seem magical-and therefore improbable - that such a recalcitrant problem as the acceptance of evolution could suddenly become much easier. Yet, the phenomenon of chemical catalysis tells us that there need be nothing magical about it, as long as we know what to do (see also Wilson 2005b; Wilson et al. 2009; O'Brien and Wilson 2010).

The first ingredient of an EvoS program at any college or university is a group of faculty who are already employing the evolutionary perspective in their own research and teaching across disciplines. The expansion beyond the biological sciences is already in full swing as far as worldwide research and scholarship are concerned. Open virtually any issue of top journals such as Science (e.g., Bowles 2009), Nature (e.g., Currie et al. 2010), or the Proceedings of the National Academy of Sciences (e.g., Goodman and Sterner 2010), and you can read about it. What is already happening at the level of science and scholarship is not yet reflected across the curriculum, but it is reflected in the research and teaching of individual faculty. Those from biology departments are accustomed to interacting with each other, but not necessarily with evolution-minded faculty from other departments. Those from human-related disciplines often feel isolated within their own departments in addition to their field as a whole. Students in any given department are largely unaware of course offerings in other departments or how such courses might satisfy their degree requirements.

An EvoS program brings evolution-minded faculty together with respect to both research and teaching. In this respect, it functions very much like a chemical catalyst, which grabs onto the component parts of the molecule that it is assembling and holds them in the right configuration so that they can combine. Even without a single additional course being taught, students can be provided with a menu of courses that were invisible to them before and a framework for taking the courses that results in a certificate, a minor, or other formal acknowledgement of their training. Once faculty start interacting with each other, they incorporate more evolution into their existing courses, start new courses, and start collaborative research. The first EvoS program, which started at Binghamton University in 2003, has become so successful in this regard that it now functions both as an undergraduate curriculum program and as an incubator for generating externally funded crossdisciplinary research. 
A key component of an EvoS program, which can also be easily established at virtually any college or university, is a campus-wide seminar series that showcases the diversity of subjects that can be studied from an evolutionary perspective. Each speaker is cohosted by the most relevant department so that faculty and graduate students from that department can learn from one of their own respected colleagues how their discipline is being approached from an evolutionary perspective. These experiences are inherently interdisciplinary. Speakers in the Binghamton and New Paltz EvoS Seminar Series have represented a plurality of academic areas including anthropology, biology, geology, philosophy, psychology - and beyond. In a single semester at New Paltz, the academic community was treated to Gordon Gallup's work on the evolutionary psychology of physical attractiveness (see Gallup and Frederick 2010), Warren Allmon's work on Darwin's influence on modern geology and biology (see Schneiderman and Allmon 2009), and David Livingstone Smith's (2009) philosophical take on warfare as it relates to human nature. And more. Lots more.

A course built around the seminar series causes undergraduate students to read and write about the primary literature, and attend seminars by scientists speaking to other scientists, and provides direct access to the speakers in the form of an extended discussion over food and drink that follows each seminar. The activities surrounding the EvoS seminar series provide a microcosm for what it means for a university to function as a single intellectual community.

In this fashion, an EvoS program can be started at modest expense and immediately begins working its catalytic magic. The Binghamton and SUNY New Paltz programs that we direct started entirely on the strength of intramural funding and quickly proved their worth in terms of both faculty and student satisfaction. In addition to a formal mechanism for teaching evolution across the curriculum, EvoS provides an informal mechanism for faculty training, especially for faculty in human-related disciplines who did not receive evolutionary training during their own higher education. In a survey given to colleagues at the forefront of studying evolution in relation to human behavior and culture, most of them reported that they did not receive formal evolutionary training and developed their expertise on their own (DSW, unpublished data). An EvoS program makes this much easier for faculty who are newly encountering the evolutionary perspective.
The catalysis that takes place on a single campus by an EvoS program can be accelerated still further by creating a consortium of programs, as we did with the help of NSF funding in 2008. It is beyond the scope of this introduction to describe the EvoS Consortium in detail, which is amply documented on the EvoS Consortium website, EvoS Journal, and the articles in this special issue of EEO. Suffice it to say that the EvoS Consortium makes it much easier to start a program and provides an impressive array of resources for member institutions and anyone who visits the website.

We believe that EvoS is the wave of the future for evolutionary training in higher education. Thanks to its ability to function as a catalyst, the wave will arrive sooner rather than later.

\section{References}

Alters BJ, Nelson CE. Perspective: teaching evolution in higher education. Evolution. 2002;56:1891-901.

Bowles S. Did warfare among ancestral hunter-gatherers affect the evolution of human social behaviors? Science. 2009;324:1293-8.

Currie TE, Greenhill SJ, Gray RD, Hasegawa T, Mace M. The rise and fall of political complexity in island SE Asia and the Pacific. Nature. 2010;476:801-4.

Dobzhansky T. Nothing in biology makes sense except in the light of evolution. Am Biol Teach. 1973;35:125-9.

Gallup GG, Frederick DA. The science of sex appeal: an evolutionary perspective. Rev Gen Psychol. 2010;14:240-50.

Gluckman P, Beedle A, Hanson M. Principles of evolutionary medicine. New York: Oxford University; 2009.

Goodman M, Sterner KN. Phylogenomic evidence of adaptive evolution in the ancestry of humans. P Natl A Sci. 2010;107 (2):8918-23.

Schneiderman JS, Allmon WD. For the rock record: geologists on intelligent design. Berkeley: University of California; 2009.

Smith DS. The most dangerous animal: human nature and the origins of war. New York: St. Martin's Griffin; 2009.

Wilson DS. Evolutionary social constructivism. In: Gottshcall J, Wilson DS, editors. The literary animal: evolution and the nature of narrative. Evanston: Northwestern University; 2005a. p. 20-37.

Wilson DS. Evolution for everyone: how to increase acceptance of, interest in, and knowledge about evolution. PLoS Biol. 2005b;3:1001-8.

Wilson DS. Evolution for everyone: how Darwin's theory can change the way we think about our lives. New York: Delacorte; 2007.

Wilson DS, Geher G, Waldo J. The EvoS consortium: completing the evolutionary synthesis in higher education. EvoS J J Evol Stud Consortium. 2009;1:3-10. 